\title{
Determining Carrier Densities in InMnAs by Cyclotron Resonance
}

\author{
G. D. Sanders ${ }^{\text {a }}$, Y. Sun ${ }^{\text {a }}$, C. J. Stanton ${ }^{\text {a }}$, G. A. Khodaparast ${ }^{\text {b }}$, J. Kono ${ }^{\text {b }}$, D. S. King ${ }^{\text {c }}$, \\ Y. H. Matsuda ${ }^{d}$, S. Ikeda ${ }^{e}$, N. Miura ${ }^{e}$, A. Oiwa $^{f}$, and H. Munekata ${ }^{\text {f }}$ \\ a Department of Physics, University of Florida \\ ${ }^{\mathrm{b}}$ Department of Electrical and Computer Engineering, Rice University \\ ${ }^{\mathrm{c}}$ Department of Applied Physics, Stanford University \\ ${ }^{\mathrm{d}}$ Department of Physics, Okayama University \\ e Institute of Solid State Physics, University of Tokyo \\ ${ }^{\mathrm{f}}$ Imaging Science and Engineering Lab, Tokyo Institute of Technology
}

\begin{abstract}
Accurate determination of carrier densities in ferromagnetic semiconductors by Hall measurements is hindered by the anomalous Hall effect, and thus alternative methods are being sought. Here, we propose that cyclotron resonance (CR) is an excellent method for carrier density determination for InMnAs-based magnetic structures. We develop a theory for electronic and magneto-optical properties in narrow gap InMnAs films and superlattices in ultrahigh magnetic fields oriented along [001]. In n-type InMnAs films and superlattices, we find that the e-active CR peak field is pinned at low electron densities and then begins to shift rapidly to higher fields above a critical electron concentration allowing the electron density to be accurately calibrated. In p-type InMnAs, we observe two h-active CR peaks due to heavy and light holes. The lineshapes depend on temperature and line broadening. The light hole CR requires higher hole densities and fields. Analyzing CR lineshapes in p-films and superlattices can help determine hole densities.
\end{abstract}

Key words: III-V magnetic semiconductors, ferromagnetism, cyclotron resonance PACS: 75.50.Pp, 78.20.Ls, 78.40.Fy

\section{Introduction}

The determination of carrier densities in semiconductors is usually carried out by Hall measurements. In ferromagnetic semiconductors such as InMnAs or GaMnAs however, the anomalous Hall effect below the Curie temperature, $T_{c}$, can often times complicate the situation and make the determination of carrier density difficult.

Recently, several alternative methods for determining the carrier densities in ferromagnetic (III,Mn) V semiconductors have been proposed. Some of these in- clude: Raman Spectroscopy,[1] C-V measurements,[2] and infrared optical conductivity. [3]

In this paper, we propose that cylcotron resonance spectroscopy can sometimes be used to very accurately determine the density for InMnAs-based magnetic structures.

While the standard method for determining density from cyclotron resonance is to integrate the total absorption, in the III-V dilute magnetic semiconductors, owing to the large amount of magnetic impurities, one must go to ultrahigh magnetic fields (40 T or more) just to be able to observe the cyclotron resonance. As 


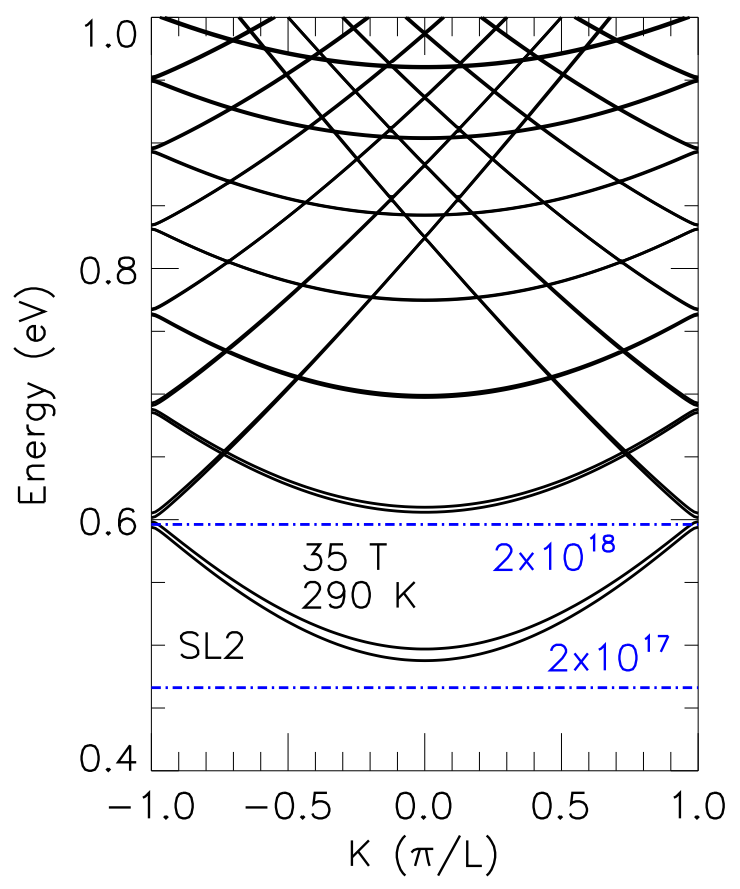

Fig. 1. Miniband structure for an n-type InAs $/ \mathrm{In}_{0.894} \mathrm{Mn}_{0.106}$ As superlattice in a 35 Tesla field at $290 \mathrm{~K}$.

a result, there is sometimes a background signal which can influence the density determined this way. Instead of using this standard method, we propose an alternative scheme based on using information which can be obtained from the CR resonant absorption such as the asymmetric lineshapes, and the positions and relative intensities of different $\mathrm{CR}$ absorption features. We show that the lineshapes, postions, relative intensities, and multiple transitions depend critically on the Fermi energy and can thus allow one to accurately determine the carrier density.

\section{2. n-type results}

We first consider InAs which is periodically doped with $\mathrm{Mn}$ to produce an n-type $\mathrm{InAs} / \mathrm{In}_{0.894} \mathrm{Mn}_{0.106} \mathrm{As}$ superlattice in which the InAs and InMnAs layers are each $50 \AA$ thick. By periodically doping with Mn, we create a spin-dependent exchange potential which tends to confine the spin-up electrons in the InAs layer and spin-down electrons in the InMnAs layer. We use

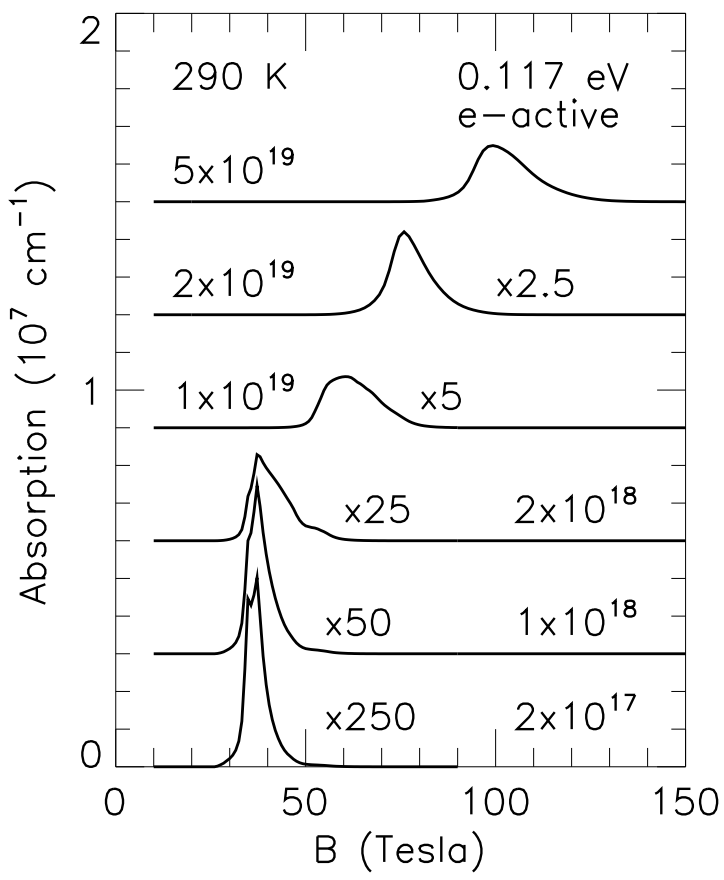

Fig. 2. Calculated room temperature CR for the n-type InAs $/ \operatorname{In}_{0.894} \mathrm{Mn}_{0.106} \mathrm{As}$ superlattice for different densities.

a modified 8 band Pidgeon-Brown model for electronic and optical properties in a high magnetic field, $B \| \hat{z}$, where $\hat{z}$ is perpendicular to the superlattice layers. The Pidgeon-Brown model [4] has been generalized to include superlattice effects due to the periodic Mn doping profile, the wavevector $\left(\mathrm{k}_{z}\right)$ dependence of the electronic states, and the position dependent $s$ - $d$ and $p$ $d$ exchange interactions with localized $\mathrm{Mn} d$-electrons. Magneto-optical properties and cyclotron resonance are obtained using Fermi's golden rule to compute the dielectric function. The Dirac delta functions appearing in the golden rule are replaced by Lorentzian line shapes with the full width at half maximum (FWHM) being an input parameter. For details, see Ref. [5]. The exchange interactions, which are parameterized by exchange integrals $\alpha$ and $\beta$, are periodic with period $\mathrm{L}$ $=100 \AA$ and give rise to minibands with $\left|k_{z}\right|<\pi / L$. The miniband structure for the superlattice at room temperature in a 35 Tesla field is shown in Fig. 1. The minibands are nearly free electron like and the zone folding of the Landau levels is clearly seen. The Fermi levels for electron concentrations of $2 \times 10^{17}$ and $2 \times 10^{18} \mathrm{~cm}^{-3}$ are shown in the figure. 
Room temperature e-active CR absorption vs. electron density, $n$, are shown in Fig. 2 for photon energies $\hbar \omega=0.117 \mathrm{eV}$ and $n$ ranging from $2 \times 10^{17}$ to $5 \times 10^{19} \mathrm{~cm}^{-3}$. The resonant field depends on carrier density. For $n<2 \times 10^{18} \mathrm{~cm}^{-3}$, the resonant field is fixed around 35 Tesla and density determination based on the CR peak position is impossible. The electronic band structure at the resonant field is shown in Fig. 1. At low carrier concentrations, CR absorption occurs between the ground state and first excited Landau levels. For $n>2 \times 10^{18} \mathrm{~cm}^{-3}$, this transition is suppressed by Pauli blocking as the first excited Landau levels start to fill. This is evident from the position of the Fermi level for $n=2 \times 10^{18} \mathrm{~cm}^{-3}$ in Fig. 1 . As $n$ continues to increase, the CR peak begins to shift rapidly to higher fields making it possible for the electron density to be accurately calibrated. If $n=10^{19} \mathrm{~cm}^{-3}$, one should be able to distinguish between $1 \times 10^{19} \mathrm{~cm}^{-3}$ and $1.1 \times 10^{19} \mathrm{~cm}^{-3}$ based on the CR peak position for an accuracy of $10 \%$ in the density. For higher $n$, the accuracy should be even better as the CR peak position shifts begins to shift more rapidly with $n$.

\section{3. p-type results}

CR measurements have been performed on p-doped InAs in h-actively polarized light with $\hbar \omega=0.117 \mathrm{eV}$ at a temperature of $27.5 \mathrm{~K}$. Experimental and theoretical CR spectra are shown in Fig. 3(a). The experimental curve is the negative of the transmission and is offset for clarity and plotted in arbitrary units. For $B>30 \mathrm{~T}$, the Fermi energy is such that only the first two Landau subbands are occupied. Transitions from these levels are responsible for the $\mathrm{CR}$ peaks labeled 1 and 2 in Fig. 3(a). The downward sloping CR absorption seen for $B<30 \mathrm{~T}$ is due to higher lying Landau levels which become populated at low fields. Only the $k=0$ Landau levels responsible for CR peaks 1 and 2 are plotted in Fig. 3(b). Resonant transitions at $0.117 \mathrm{eV}$ are indicated by vertical lines. The CR peak, 2, near $40 \mathrm{~T}$ is due to transitions between the spindown ground-state heavy hole Landau subband, $H_{-1,1}$ with energy $E_{-1}\left(k_{z}\right)$ and the heavy-hole subband, $H_{0,2}$ with energy $E_{0}\left(k_{z}\right)$. The CR peak, 1 , seen at higher fields, is a spin-down light-hole transition between the $L_{0,3}$ and $L_{1,4}$ subbands. The two CR peaks are seen to
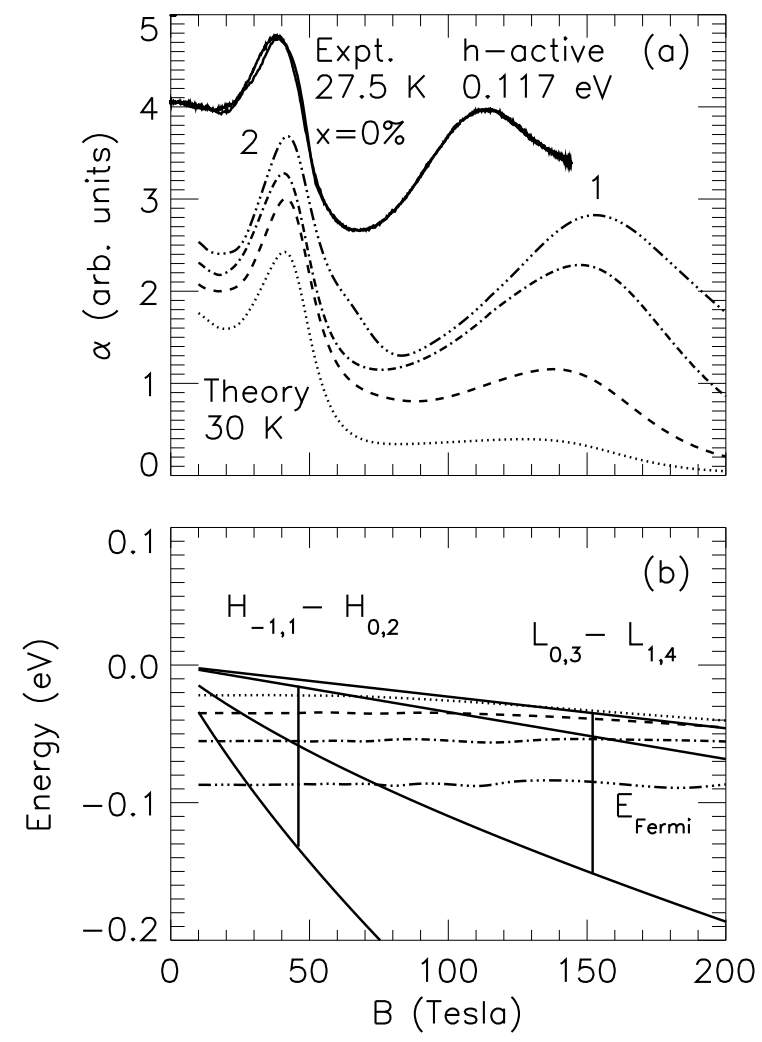

Fig. 3. Experimental and theoretical h-active CR for p-type InAs (a). Theoretical CR curves, from bottom to top, assume hole densities of $5 \times 10^{18}, 10^{19}, 2 \times 10^{19}$, and $4 \times 10^{19} \mathrm{~cm}^{-3}$. In (b), Landau levels involved in observed CR peaks are shown along with Fermi levels corresponding to theoretical curves in (a).

be asymmetric about their respective resonance fields. While the theoretical light hole peak does not fit the experimental peak position, by varying the Luttinger parameter $\gamma_{1}$, the peak position can be brought into better agreement with experiment. This shows that CR can be used to determine band parameters and effective masses. The relative strengths of the heavy and light-hole CR peaks is sensitive to the itinerant hole density and can be used to determine the hole density. By comparing theoretical and experimental curves in Fig. 3(a), we see that the itinerant hole concentration is around $2 \times 10^{19} \mathrm{~cm}^{-3}$. From Fig. 3(a), we can rule out $n<10^{19} \mathrm{~cm}^{-3}$ and $n>4 \times 10^{19} \mathrm{~cm}^{-3}$. We estimate that an error in the hole density of around $25 \%$ should be achievable at these densities.

We next consider the effects of Mn doping on CR in 

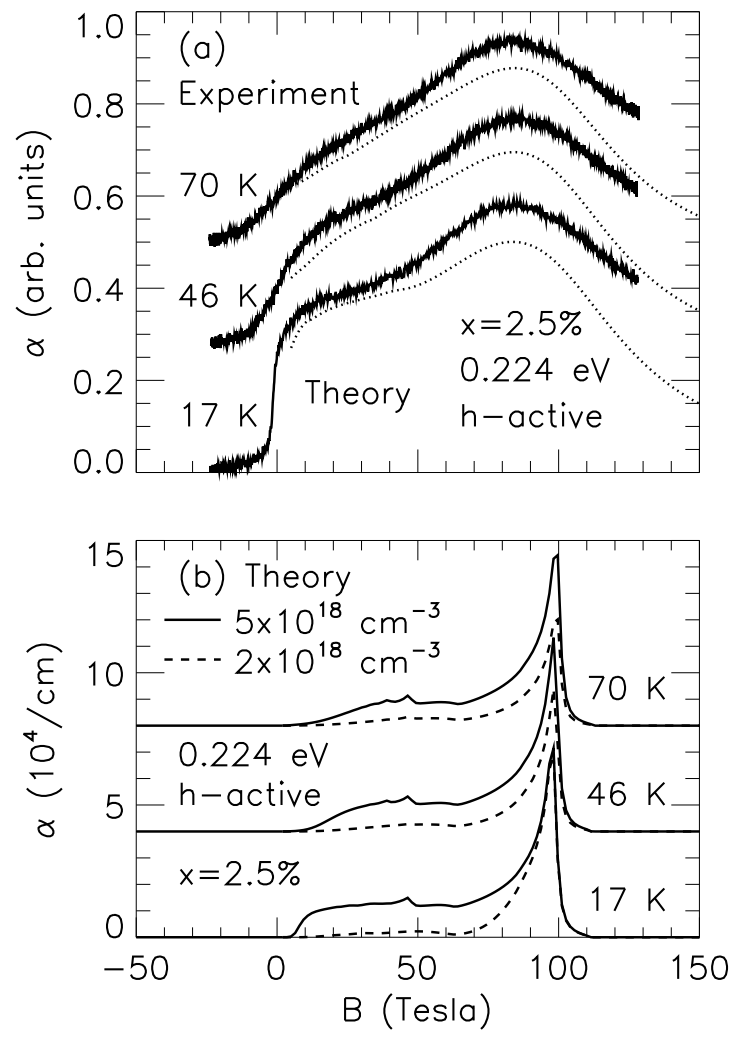

Fig. 4. CRs for p-type $\operatorname{In}_{0.975} \mathrm{Mn}_{0.025}$ As. Experimental and theoretical CRs are shown in (a) for several temperatures. In (b), theoretical CRs are shown for two hole densities.

InAs. We performed CR on p-type $\operatorname{In}_{0.975} \mathrm{Mn}_{0.025} \mathrm{As}$ at temperatures of 17,46 , and $70 \mathrm{~K}$ in h-active circularly polarized light with $\hbar \omega=0.224 \mathrm{eV}$. It is more convenient to perform $\mathrm{CR}$ at higher photon energies, and hence higher resonant fields, in order to avoid the low field tails seen in Fig. 3(a) for $B<30$ T. This allows us to better examine the lineshapes. In Fig. 4(a), the experimental CR is shown as a function of the magnetic field for the three temperatures and the theoretical CR spectra are shown as dotted lines. In the theoretical curves, the FWHM linewidths are taken to be $120 \mathrm{meV}$ and the hole concentration is taken to be $p=$ $5 \times 10^{18} \mathrm{~cm}^{-3}$. A heavy hole CR transition is seen at a resonant field near 80 Tesla. The resonant field is insensitive to temperature and the lineshape is strongly asymmetric with a broad tail at low fields. The width of the low field tail depends on the hole conentration as seen in Fig. 4(b) where theoretical CR are computed at two different hole condentrations assuming a FWHM of $4 \mathrm{meV}$. The low field tail is thus a sensitive probe of the hole density. The sharpness of the low field cutoff depends on temperature and can be attributed to the sharpness of the Fermi distribution at low temperatures. In this sample, our estimate of the hole concentration not all that accurate, but we can see the hole concentration is greater than $2 \times 10^{18} \mathrm{~cm}^{-3}$ based on the lack of a low field tail in the latter case.

\section{Conclusions}

We have developed a theory for electronic and magneto-optical properties in narrow gap InMnAs films and superlattices in ultrahigh magnetic fields and have shown the $\mathrm{CR}$ can be a valuable tool for determining the densities of itinerant carriers in these systems. In n-type InMnAs films and superlattices, we found that the e-active CR peak field is pinned at low electron densities and then begins to shift rapidly to higher fields above a critical electron concentration allowing the electron density to be accurately determined. In p-type materials, determination of hole densities from CR measurements is more involved. In p-type InMnAs, we observed two h-active CR peaks due to heavy and light holes. The lineshapes were found to depend on temperature and line broadening. Analyzing CR lineshapes in p-films and superlatices can help determine hole densities.

We gratefully acknowledge support from NSF DMR9817828, DMR-0134058 (CAREER), and DARPA MDA972-00-1-0034 (SPINS).

\section{References}

[1] M. J. Seong, S. H. Chun, H. M. Cheong, N. Samarth, and A. Mascarenhas Phys. Rev. B 66033202 (2002).

[2] R. Moriya, and H. Munekata, J. Appl. Phys. 93, 4603 (2003).

[3] J. Sinova, T. Jungwirth, S.-R. Eric Yang, J. Kuc̈era, and A. H. MacDonald, Phys. Rev. B 66, 041202 (2002).

[4] C. K. Pidgeon and R. N. Brown, Phys. Rev. 146, 575 (1966).

[5] G. D. Sanders, Y. Sun, F. V. Kyrychenko, C. J. Stanton, G. A. Khodaparast, M. A. Zudov, J. 
Kono, Y. H. Matsuda, N. Miura, and H. Munekata, cond-mat/0304434 